Research Article

\title{
Around the Lipschitz Summation Formula
}

\author{
Wenbin Li, ${ }^{1}$ Hongyu Li $\mathbb{D}^{1},{ }^{1}$ and Jay Mehta $\mathbb{D}^{2}$ \\ ${ }^{1}$ Suda Science \& Technology Research Institute, Sanmenxia, Henan 472000, China
}

${ }^{2}$ Department of Mathematics, Sardar Patel University, Vallabh Vidyanagar 388 120, Anand, India

Correspondence should be addressed to Jay Mehta; jaygmehta@gmail.com

Received 15 January 2020; Accepted 5 March 2020; Published 23 April 2020

Guest Editor: Praveen Agarwal

Copyright (c) 2020 Wenbin Li et al. This is an open access article distributed under the Creative Commons Attribution License, which permits unrestricted use, distribution, and reproduction in any medium, provided the original work is properly cited.

Boundary behavior of important functions has been an object of intensive research since the time of Riemann. Kurokawa, Kurokawa-Koyama, and Chapman studied the boundary behavior of generalized Eisenstein series which falls into this category. The underlying principle is the use of the Lipschitz summation formula. Our purpose is to show that it is a form of the functional equation for the Lipschitz-Lerch transcendent (and in the long run, it is equivalent to that for the Riemann zeta-function) and that this being indeed a boundary function of the Hurwitz-Lerch zeta-function, one can extract essential information. We also elucidate the relation between Ramanujan's formula and automorphy of Eisenstein series.

\section{Introduction}

Boundary behavior of core functions has always been the object of intensive research since it exhibits a peculiar phenomenon that cannot be predicted by the behavior inside the domain. There are many instances of such unexpected behavior cf. [1-3]. Kurokawa [4] and Koyama and Kurokawa [5] studied the following limiting values by the Lipschitz summation formula:

$$
\lim _{\tau \longrightarrow x}\left(E_{k}\left(-\frac{1}{\tau}\right)-\tau^{k} E_{k}(\tau)\right), \quad \forall x \in \mathbb{R},
$$

where $E_{k}(\tau)$ is the generalized Eisenstein series defined by (25).

It has been elucidated and generalized by Chapman [6] who also used the Lipschitz summation formula for which he appealed to [7]. Knopp and Robbins in their Remarks 1 and 2 state their own views on the Lipschitz summation formula and Stark's method [8] to the effect that they are not directly related to the functional equation (just as, for the Riemann zeta-function, the partial fraction expansion does not seem to be related). In [9], Murty and Sinha [10] result has been elucidated as a manifestation of one of the equivalent conditions to the functional equation, the Fourier-Bessel expansion, or the perturbed Dirichlet series ([11], Chapter 4), thereby explaining the genesis of Stark's method. The Lipschitz summation formula for quadratic fields is also deduced there. We shall turn to this toward the end of Section 4.

We cite the passage from [12] "The relation between modular forms and Dirichlet series with functional equations was discovered by Hecke, whose epoch-making work during the years 1930-1940, based on that discovery and that of the 'Hecke operators', brought out completely new aspects of a theory which many mathematicians would have regarded as a closed chapter long before."

We refer to this as part of the Riemann-Hecke-Bochner correspondence (RHB correspondence) ([11], p. 4 and 22) which is coined by Knopp [13].

Our main aim in this paper is to prove the general modular relation, Theorem 4, for the Lipschitz-Lerch transcendent (57) and deduce the general Lipschitz summation formula, Corollary 4 . From this, we show that, in this case again, generalized RHB correspondence or the modular relation is the key for everything.

But prior to this, in Section 2, we state the modular relation for the Lambert series generated by the product of two Riemann zeta-functions with variables different by an odd integer and prove the automorphy of the Eisenstein series by the RHB correspondence, which of course settles the even weight case of (1). For another relation, cf. Bruinier and Funke [14]. 
The even difference case turns out to be a reminiscent of the Wigert-Bellman divisor problem [15] as alluded to in [9]. In Section 3, we state the results in another form based on the shifted Mellin inversion.

Here, we use a method similar to the one in [16] (pp. 73-75) of using the Ewald expansion ([11], Chapter 5) as opposed to the Fourier-Bessel expansion alluded to above. Since it is equivalent to the Lerch functional equation ([17], Theorem 5.3, p. 130) which in turn is equivalent to an asymmetric form (3) of the functional equation for the Riemann zeta-function, we thereby show that, in the long run, the genesis is in the functional equation for the Riemann zeta-function.

As a necessary step, we show that the reciprocal Hurwitz formula amounts to a ramified functional equation, Lemma 1. There are many cases of such ramified functional equations (cf. [18] and references therein). We state one of the earliest occurrences.

In what follows, we always use the notation $s=\sigma+i t$ as the complex variable.

\section{An Example of the}

\section{Riemann-Hecke-Bochner Correspondence}

Throughout in what follows, we appeal to the Riemann zetafunction defined in the first instance for $\sigma=\operatorname{Res}>1$ by

$$
\zeta(s)=\prod_{p}\left(1-\frac{1}{p^{s}}\right)^{-1}=\sum_{n=1}^{\infty} \frac{1}{n^{s}} .
$$

This satisfies the asymmetric form of the functional equation:

$$
\zeta(1-s)=2(2 \pi)^{-s} \cos \left(\frac{\pi}{2} s\right) \Gamma(s) \zeta(s),
$$

which is a prototype of the Hurwitz formula (cf. (64) for its reciprocal).

We fix the integer $\alpha$ throughout.

We consider the product of two zeta-functions:

$$
\varphi(s)=\varphi(s, \alpha)=\zeta(s) \zeta(s+\alpha)=\sum_{n=1}^{\infty} \frac{\sigma_{-\alpha}(n)}{n^{s}},
$$

where the series is absolutely convergent for $\sigma>\sigma_{\varphi}:=\max \{1,1-\alpha\}$, and

$$
\sigma_{-\alpha}(n)=\sum_{d \mid n} d^{-\alpha}
$$

is the sum-of-divisors function. We note that $\varphi(s-a)$ includes the case of $\zeta(s) \zeta(s-\alpha)$ as $\varphi(s-a)=\sum_{n=1}^{\infty}\left(\left(\sigma_{\alpha}(n)\right) / n^{s}\right)$. This will be pursued in Section 3.

The zeta-function $\varphi(s)$ satisfies the asymmetric functional equation:

$$
\varphi(1-s)=4(2 \pi)^{-2 s+\alpha} \cos \frac{\pi}{2} s \cos \frac{\pi}{2}(s-\alpha) \Gamma(s) \Gamma(s-\alpha) \varphi(s-\alpha) .
$$

$$
\cos \frac{\pi}{2}(s-\alpha)= \begin{cases}\sin \frac{\pi}{2} s \sin \frac{\pi}{2} \alpha, & \alpha=2 \varkappa+1, \\ \cos \frac{\pi}{2} s \cos \frac{\pi}{2} \alpha, & \alpha=2 \varkappa,\end{cases}
$$

the product of cosines amounts to

$$
\begin{cases}\frac{1}{2}(-1)^{\varkappa} \sin \pi s, & \alpha=2 \varkappa+1, \\ \frac{1}{2}(-1)^{\varkappa}(1+\cos \pi s), & \alpha=2 \varkappa .\end{cases}
$$

(i) First we treat the case of $\alpha=2 \varkappa+1$ an odd integer. Then by the reciprocal relation for the gamma function, we see that the functional equation (6) amounts to

$$
\varphi(1-s)=2(-1)^{\varkappa}(2 \pi)^{-2 s+\alpha} \frac{\pi}{\Gamma(1-s)} \Gamma(s-\alpha) \varphi(s-\alpha) .
$$

Now by the well-known procedure-Hecke gamma transform (e.g., [16]), we have for $c>\sigma_{\varphi}$ and $\operatorname{Re} x>0$

$$
\sum_{n=1}^{\infty} \sigma_{-\alpha}(n) e^{-n x}=\frac{1}{2 \pi i} \int_{(c)} \Gamma(s) \varphi(s) x^{-s} \mathrm{~d} s
$$

where $(c)$ indicates the Bromwich contour $\sigma=c,-\infty<t<\infty$.

By a standard procedure of moving the line to the left up to $(d)$, where $d<-\alpha<0(d=-\alpha-(1 / 2)$, say), whereby noting that the horizontal integrals vanish in the limit as $|t| \longrightarrow \infty$, we obtain

$$
\frac{1}{2 \pi i} \int_{(c)} \Gamma(s) \varphi(s) x^{-s} \mathrm{~d} s=\frac{1}{2 \pi i} \int_{(d)} \Gamma(s) \varphi(s) x^{-s} \mathrm{~d} s+P(x),
$$

where $P(x)=P_{\alpha}(x)$ is the residual function consisting of the sum of residues of the integrand at $-a, \ldots,-1,0,1, a+1$. Writing $1-s$ for $s$ in (11), we see that the right-hand side of (11) becomes $(1 / 2 \pi i) \int_{(1-d)} \Gamma(1-s) \varphi(1-s) x^{s-1} \mathrm{~d} s+P(x), \quad$ whence substituting (9), we conclude that

$$
\begin{aligned}
\sum_{n=1}^{\infty} \sigma_{-\alpha}(n) e^{-n x}= & (-1)^{x}(2 \pi)^{\alpha+1} \frac{1}{x} \frac{1}{2 \pi i} \\
& \cdot \int_{(1-d)} \Gamma(s-\alpha) \varphi(s-\alpha)\left(\frac{4 \pi^{2}}{x}\right)^{-s} \mathrm{~d} s+P(x) .
\end{aligned}
$$


Substituting the absolutely convergent series in (4) and factoring out $\left(x / 4 \pi^{2}\right)^{a}$, we deduce that

$$
\begin{aligned}
\sum_{n=1}^{\infty} \sigma_{-\alpha}(n) e^{-n x}= & (-1)^{\varkappa}(2 \pi)^{\alpha+1} \frac{1}{x}\left(\frac{x}{4 \pi^{2}}\right)^{\alpha} \sum_{n=1}^{\infty} \sigma_{-\alpha}(n) \\
& \times \frac{1}{2 \pi i} \int_{(1-d)} \Gamma(s-\alpha)\left(\frac{4 \pi^{2} n}{x}\right)^{-(s+\alpha)} \mathrm{d} s+P(x) .
\end{aligned}
$$

Hence, by the Mellin inversion again, we obtain

$$
\sum_{n=1}^{\infty} \sigma_{-\alpha}(n) e^{-n x}=(-1)^{(\alpha-1) / 2}\left(\frac{x}{2 \pi}\right)^{\alpha-1} \sum_{n=1}^{\infty} \sigma_{-\alpha}(n) e^{-\left(4 \pi^{2} n\right) / x}+P(x),
$$

for $\operatorname{Re} x>0$, i.e., the Bochner modular relation [19].

Here only in the case $\alpha=1>0, s=1$ is a double pole, others being simple poles. In the case $\alpha=-1<0$, there is one more term $-(1 / 2) x^{-1}$.

To compute the residual function, we use Table 1, taking into account the trivial zeros of the Riemann zeta-function at negative even integers.

For $1 \neq \alpha>0$, the residual function is

$$
\begin{aligned}
P_{\alpha}(x)= & \sum_{k=1}^{\alpha} \frac{(-1)^{k}}{k !} \zeta(-k) \zeta(-k+\alpha) x^{k}+\zeta(1+\alpha) x^{-1}-\frac{1}{2} \zeta(\alpha) \\
= & -\sum_{j=1}^{[\alpha / 2]+1} \frac{1}{(2 j-1) !} \zeta(1-2 j) \zeta(\alpha-2 j+1) x^{2 j-1} \\
& +\zeta(1+\alpha) x^{-1}-\frac{1}{2} \zeta(\alpha),
\end{aligned}
$$

on writing $k=2 j-1$. In literature, this is expressed in another form based on the explicit formula for zetavalues cf., e.g., ([20], p. 71 and 91):

$$
\zeta(1-2 k)=-\frac{B_{2 k}}{2 k} \quad(k \geq 1), \quad \zeta(2 k)=\frac{2^{2 k-1}}{(2 k) !} B_{2 k} \pi^{2 k},
$$

where the Bernoulli numbers $B_{2 k}$ are $b$-notation ([20], p. 90).

In particular, for $\alpha=2 \varkappa+1 \geq 1$, (14) with $x$ replaced by $2 \pi x$ amounts to the celebrated Ramanujan formula, cf., e.g., [21] for a general account:

$$
\sum_{n=1}^{\infty} \sigma_{-2 \varkappa-1}(n) e^{-2 \pi n x}+(-1)^{\varkappa+1} x^{2 \varkappa} \sum_{n=1}^{\infty} \sigma_{-2 \varkappa-1}(n) e^{-((2 \pi n) / x)}=P(x),
$$

where $P(x)=P_{2 \varkappa+1}(x)$ is given by (15) whose concrete form is given in (49). Indeed, $P_{2 \varkappa+1}(x)$ is a residual function given as the sum of the residues:

$P(x)=\sum_{\xi \in R} \operatorname{Res}_{s=\xi}(2 \pi)^{-s} \Gamma(s) \zeta(s) \zeta(s+2 \varkappa+1) x^{-s}$,

where $R=\{-2 \varkappa-1,-2 \varkappa,-2 \varkappa+1,-2 \varkappa+3, \ldots,-3,-$ $1,0,1\}$, and $s=0$ is a double pole only when $\varkappa=0$ (others are simple poles). Hence,

$$
\begin{aligned}
P_{2 \varkappa+1}(x)= & \sum_{j=1}^{\varkappa+1} \frac{(-1)^{k}}{(2 j+1) !} \zeta(1-2 j) \zeta(2 \varkappa-2 j+2) x^{2 j-1} \\
& +\zeta(2 \varkappa+2) x^{-1}-\frac{1}{2} \zeta(2 \varkappa+1) .
\end{aligned}
$$

cf. (49) below for another expression for the residual function.

If we write $-x=2 \pi i \tau$, then $\tau \in \mathscr{H}$ and the Lambert series in Liouville's form amounts to the Eisenstein series $E_{k}(\tau)$ in Definition 1, where $k=a+1$ is even and (14) gives

$$
\sum_{n=1}^{\infty} \sigma_{-\alpha}(n) e^{2 \pi i n \tau}=\tau^{\alpha-1} \sum_{n=1}^{\infty} \sigma_{-\alpha}(n) e^{-((2 \pi i n) / \tau)}+P(x) .
$$

In case $\alpha=-2 \varkappa-1, \varkappa \geq 1,(20)$ reads

$$
\begin{aligned}
\sum_{n=1}^{\infty} \sigma_{2 \varkappa+1}(n) e^{2 \pi i n \tau}= & \tau^{\alpha-1} \sum_{n=1}^{\infty} \sigma_{2 \varkappa+1}(n) e^{-((2 \pi i n) / \tau)}+P(x), \\
P(\tau)= & P_{-2 \varkappa-1}(\tau)=(2 \varkappa+1) ! \zeta(2 \varkappa+2) \\
& \cdot(-2 \pi i \tau)^{-2 \varkappa-2}-\frac{1}{2} \zeta(-2 \varkappa-1),
\end{aligned}
$$

cf. (51) and (52).

In slightly different notation from [20] (p. 83),

$$
G_{2 k}(\tau):=\sum_{\substack{m, n \in \mathbb{Z} \\(m, n) \neq(0,0)}} \frac{1}{(n \tau+m)^{2 k}}
$$


By Proposition 4 in [20] (p. 83), $G_{2 k}(\tau)$ is a modular form of weight $2 k$. The Laurent expansion (or $q$-expansion) reads ([20], p. 92)

$$
G_{2 k}(\tau)=2 \zeta(2 k)+\frac{2(2 \pi i)^{2 k}}{(2 k-1) !} \sum_{n=1}^{\infty} \sigma_{2 k-1}(n) e^{2 \pi i n \tau}, \quad \tau \in \mathscr{H}
$$

Appealing to (16), we have similarly to [20] (Corollary, p. 92)

$\frac{1}{2 \zeta(2 k)} G_{2 k}(\tau)=\frac{2}{\zeta(1-2 k)} E_{2 k}(\tau)=-\frac{2 k}{B_{2 k}} E_{2 k}(\tau)$,

where $(2 /(\zeta(1-2 k))) E_{2 k}(\tau)$ is the Eisenstein series ([20], (34), p. 92) and $E_{2 k}(\tau)$ is the even suffix case of the following.

Definition 1. For any $k \in \mathbb{N}$, Kurokawa introduces the general Eisenstein series:

$$
E_{k}(\tau)=\frac{\zeta(1-k)}{2}+\sum_{n=1}^{\infty} \sigma_{k-1}(n) e^{2 \pi i n \tau}, \quad \tau \in \mathscr{H}
$$

which is not necessarily modular for $k$ odd.

Stating (21) in explicit form

$$
\begin{aligned}
& \sum_{k=1}^{\infty} \sigma_{2 \varkappa+1}(k) e^{-2 \pi k x}-\frac{B_{2 \varkappa+2}}{4 \varkappa+4} \\
& \quad=(-1)^{\varkappa+1} x^{-2 \varkappa-2}\left\{\sum_{k=1}^{\infty} \sigma_{2 \varkappa+1}(k) e^{-((2 \pi k) / x)}-\frac{B_{2 \varkappa+2}}{4 \varkappa+4}\right\},
\end{aligned}
$$

we see that it is nothing but

$$
E_{2 \varkappa+2}\left(-\frac{1}{\tau}\right)=\tau^{2 \varkappa+2} E_{2 \varkappa+2}(\tau)
$$

i.e., the automorphy of $E_{2 \varkappa+2}(z)$, cf. (25).

Thus, we have established.

Theorem 1. The Bochner modular relation (14) entails at one end of the spectrum $\alpha=-(2 \varkappa+1)$ Ramanujan's formula (17) and at the other end $\alpha=2 \varkappa+1$ the automorphy of the Eisenstein series (27), thus abridging analytic number theory and the theory of modular forms.

(ii) Now we turn to the case of $a=2 \varkappa$. We digress from (12) which should be replaced by

$$
\begin{aligned}
\sum_{n=1}^{\infty} \sigma_{a}(n) e^{-n x}= & 2(-1)^{\varkappa}(2 \pi)^{-2 s+a} \frac{\pi}{x} \frac{1}{2 \pi i} \int_{(1-d)} \frac{\pi}{\sin \pi s} \Gamma \\
& \cdot(s-a) \varphi(s)\left(\frac{4 \pi^{2} n}{x}\right)^{-s} \mathrm{~d} s \\
& +2(-1)^{\varkappa}(2 \pi)^{-2 s+a} \frac{\pi}{x} \frac{1}{2 \pi i} \int_{(1-d)} \pi \cot \pi s \Gamma \\
& \cdot(s-a) \varphi(s)\left(\frac{4 \pi^{2} n}{x}\right)^{-s} \mathrm{~d} s+P(x) .
\end{aligned}
$$

In the same way as we have deduced (13), we obtain

$$
\begin{aligned}
\sum_{n=1}^{\infty} \sigma_{a}(n) e^{-n x}= & 2(-1)^{\varkappa}(2 \pi)^{-2 s+a} \frac{\pi}{x} \frac{1}{2 \pi i} \int_{(1-d)} \frac{\pi}{\sin \pi s} \Gamma \\
& \cdot(s-a) \varphi(s)\left(\frac{4 \pi^{2} n}{x}\right)^{-s} \mathrm{~d} s \\
& +2(-1)^{\varkappa}(2 \pi)^{-2 s+a} \frac{\pi}{x} \frac{1}{2 \pi i} \int_{(1-d)} \pi \cot \pi s \Gamma \\
& \cdot(s-a) \varphi(s)\left(\frac{4 \pi^{2} n}{x}\right)^{-s} \mathrm{~d} s+P(x) .
\end{aligned}
$$

We shall stop here since it would be difficult to express the resulting integrals and Bellman's method [15] yields an asymptotic formula rather than an equality. Partial theory of modular relations for the product of zetafunctions is given in ([11], Chapter 9, pp. 241-265), which is still in progress.

Remark 1. That the odd integer difference case (i) reduces to the one-gamma factor case to the RHB correspondence is not coincidental and is expounded in [11] (pp. 81-86), where one can also find a plausible discovery of Ramanujan of the transformation formula for the Dedekind eta-function $\eta$. Weil's paper [22] is the most well-known paper that contains the proof of the latter, but prior to this, Chowla gave a proof [23] for the discriminant function, which is the 24th power of $\eta$. Ramanujan's formula is stated as I, Entry 15 of Chapter 16 [24], Entry 21 (i), Chapter 14 of Ramanujan's Notebook II [25] (which is and also as IV, Entry 20 of [26]). The most extensive account of information surrounding Ramanujan's formula is [27], while [28] is the most informative account of special values of the zetafunctions. The intersection of references in these two excellent survey papers (which have a lot in common) is a null set. In literature, Ramanujan's formula is stated for $\alpha=\pi x>0, \beta=$ $(\pi / x)>0$ satisfying the following relation: 


$$
\alpha \beta=\pi^{2},
$$

and in terms of Lambert series.

The Lambert series $L(z)$ is defined for $|z|<1$ by

$$
L(z)=\sum_{n=1}^{\infty} a_{n} \frac{z^{n}}{1-z^{n}}
$$

which is transformed into the Fourier series:

$$
L(z)=\sum_{m=1}^{\infty} \sum_{n=1}^{\infty} a_{n}\left(z^{m}\right)^{n}=\sum_{\ell=1}^{\infty} b_{\ell} z^{\ell},
$$

the Liouville formula, where $b_{\ell}=\sum_{d \mid \ell} a_{d}$. The sum-of-divisors function is the case $a_{n}=n^{\alpha}$. Original Ramanujan's formula looks like having little to do with modular forms. Equation (17) being a rephrased Lambert series in Liouville's form has amenity to the $q$-expansion, and so to automorphy.

\section{The Ramanujan-Guinand Formula and Its Consequences}

In Section 2, we established that at both ends of the spectrum, the Bochner modular relation amounts to Ramanujan's formula and the automorphy of Eisenstein series, respectively. In this section, we partially follow [29], reproduced in [11] (pp. 86-92), and elucidate the mechanism hidden in the correspondence $\varphi(s) \longleftrightarrow \varphi(s-a)$ by differentiation of the Ramanujan-Guinand formula.

In [14], they mention duality between the space of weak Maass forms of (negative) weight $k \in(1 / 2) \mathbb{Z}$ and the space of holomorphic cusp form of (positive) weight $2-k$.

To this end, we introduce the Mellin inversion with shifted argument $I_{a}(x)$. Let $a \geq 0$ be a fixed integer to be taken as the number of times of differentiation throughout. Let $\varphi(s)$ be the zeta-function defined by (4) with $\alpha=2 \varkappa+1$ and $\varkappa$ a nonnegative integer:

$$
\varphi(s)=\zeta(s) \zeta(s+2 \varkappa+1),
$$

the other case being included in Theorem 2 below. The argument of its proof goes in the lines of Section 2. For Rex $>0$ let

$$
I_{a}(x)=I_{a, \varkappa}(x)=\frac{1}{2 \pi i} \int_{(c)}(2 \pi)^{-s} \Gamma(s) \varphi(s-a) x^{-s} \mathrm{~d} s,
$$

where $c>1+a$. The Hecke gamma transform reads

$$
I_{a}(x)=\sum_{k=1}^{\infty} \sigma_{-2 \varkappa-1}(k) k^{a} e^{-2 \pi k x} .
$$

The special case,

$$
I(x)=I_{0}(x)=\frac{1}{2 \pi i} \int_{(k)}(2 \pi)^{-s} \Gamma(s) \varphi(s) x^{-s} \mathrm{~d} s=\sum_{k=1}^{\infty} \sigma_{-2 \varkappa-1}(k) e^{-2 \pi k x},
$$

is the Lambert series appearing in Ramanujan's formula (17). Differentiating $I(x) a$-times with respect to $x$, whereby we perform differentiation under integral sign, we have the additional factor

$$
\prod_{j=0}^{a-1}(-s-j)
$$

which is $(-1)^{a}(\Gamma(s+a) / \Gamma(s))$, whence we deduce the remarkable formula:

$$
\frac{\mathrm{d}^{a}}{\mathrm{~d} x^{a}} I(x)=(-2 \pi)^{a} I_{a}(x),
$$

i.e., $a$-times differentiation of the Lambert series (36) is effected by shifting the argument of $\varphi(s)$ by $a$ in (36) and multiplying by $(-2 \pi)^{a}$. In view of (38), the $a$-times differentiated form of Ramanujan's formula (17) amounts to a counterpart of the modular relation for $I_{a}(x)$.

Theorem 2 (Ramanujan-Guinand formula). For the Mellin transform $I_{a}(x)$ with shifted argument as defined by (34), we have the modular relation for $x \geq 0$ and $0 \leq a \leq 2 x+1$,

$$
\begin{aligned}
I_{a}(x)= & (-1)^{x+a}(2 \pi)^{-a} x^{-a+2 x} \sum_{k=0}^{a}\left(\begin{array}{l}
a \\
k
\end{array}\right) \frac{(2 x-k) !}{(2 x-a) !} \\
& \cdot\left(\frac{2 \pi}{x}\right)^{k} I_{k}\left(\frac{1}{x}\right)+P_{a}(x),
\end{aligned}
$$

where $P_{a}(x)$ is the residual function.

Proof. Proof depends on the following equation:

$$
\varphi(s-a)=(-1)^{n}(2 \pi)^{2 \varkappa+2 s-2 a} \frac{\Gamma(a-2 \varkappa-s)}{\Gamma(s-a)} \varphi(a-2 \varkappa-s),
$$

which is a variant of the functional equation (6) in the following form:

$$
(2 \pi)^{-s} \Gamma(s) \varphi(s)=(-1)^{n}(2 \pi)^{2 \varkappa+s} \Gamma(-2 \varkappa-s) \varphi(-2 \varkappa-s) .
$$

Moving the line of integration to $\sigma=-c_{1}\left(c_{1}>2 \varkappa+1-a\right)$, we have

$$
\begin{aligned}
& I_{a}(x)=J_{a}(x)+P_{a}(x), \\
& J_{a}(x)=\frac{1}{2 \pi i} \int_{\left(-c_{1}\right)} \Gamma(s) \varphi(s-a)(2 \pi x)^{-s} \mathrm{~d} s,
\end{aligned}
$$

where $P(x)=P_{a}(x)$ denotes the sum of residues of the integrand at its poles at $s=a-2 \varkappa-1, a-2 \varkappa, a-2 \varkappa+1, a-2 \varkappa+3, \ldots, 0, a+1$.

Substitute (40) and change the variable $s \longleftrightarrow a-2 \chi-s$ in the integral $J_{a}(x)$.

Then

$$
J_{a}(x)=\frac{(-1)^{\varkappa}}{2 \pi i} \int_{\left(a-2 \varkappa+c_{1}\right)} \frac{\Gamma(a-2 \varkappa-s) \Gamma(s)}{\Gamma(-2 \varkappa-s)} \varphi(s)(2 \pi)^{-s-a} x^{s-a+2 \varkappa} \mathrm{d} s .
$$

Substituting 
TABLE 1: Poles and the sum of residues in the relevant domain.

\begin{tabular}{ccccc}
\hline Case & $\sigma_{\varphi}$ & $d$ & Poles & $P_{\alpha}(x)$ \\
\hline$\alpha>0$ & 1 & $d=\alpha-(1 / 2)$ & $-\alpha, \ldots,-1,0,1$ & $(2.12)$ \\
$\alpha<0$ & $1-\alpha$ & $d=-(1 / 2)$ & $0,1,1-\alpha$ & $\Gamma(1-\alpha) \zeta(1-\alpha) x^{\alpha-1}-(1 / 2) \zeta(\alpha)$ \\
\hline
\end{tabular}

$$
\begin{aligned}
\frac{\Gamma(a-2 \varkappa-s)}{\Gamma(-2 \varkappa-s)} & =\frac{\Gamma(1+s+2 \varkappa)}{\Gamma(1+s+2 \varkappa-a)} \frac{\sin \pi s}{\sin \pi(s-a)} \\
& =(-1)^{a} \frac{\Gamma(a+2 \varkappa+1)}{\Gamma(s+2 \varkappa+1-a)},
\end{aligned}
$$

we find that

$$
J_{a}(x)=\frac{(-1)^{\varkappa+a}}{2 \pi i} \int_{\left(a-2 \varkappa+c_{1}\right)} \frac{\Gamma(s+2 m \varkappa+1) \Gamma(s)}{\Gamma(s+2 \varkappa+1-a)} \varphi(s)(2 \pi)^{-s-a} x^{s-a+2 \varkappa} \mathrm{d} s .
$$

Since the gamma factor can be computed as follows for $0 \leq a \leq 2 \varkappa+1$,

$$
\frac{\Gamma(s) \Gamma(s+2 \varkappa+1)}{\Gamma(s+2 \varkappa+1-a)}=\sum_{k=0}^{a}\left(\begin{array}{l}
a \\
k
\end{array}\right) \frac{(2 \varkappa-k) !}{(2 \varkappa-a) !} \Gamma(s+k)
$$

where for $a=2 \varkappa+1$, the right-hand side is to mean $\Gamma(s+2 \varkappa+1)$, we conclude from $(42)$ and $(45)$ that

$$
\begin{aligned}
I_{a}(x)= & (-1)^{\varkappa+a}(2 \pi)^{-a} x^{-a+2 \varkappa} \sum_{k=0}^{a}\left(\begin{array}{l}
a \\
k
\end{array}\right) \frac{(2 \varkappa-k) !}{(2 \varkappa-a) !} \\
& \times \frac{1}{2 \pi i} \int_{\left(a-2 \varkappa+c_{1}\right)} \Gamma(s+k) \varphi(s)\left(\frac{2 \pi}{x}\right)^{-s} \mathrm{~d} s+P_{a}(x),
\end{aligned}
$$

where the sum reduces to 1 for $a-2 \varkappa+1$. Finally, we note that the integral on the right-hand side of (47) becomes by the change of variable $s \longleftrightarrow s+k$

$$
\left(\frac{2 \pi}{x}\right)^{k} I_{k}\left(\frac{1}{x}\right)
$$

Hence, (47) leads to (39), completing the proof.

\section{Corollary 1}

(i) The case $a=0$ is Ramanujan's formula (17) with the residual function

$$
\begin{aligned}
P(x)= & \frac{(2 \pi)^{2 \varkappa+1}}{2 x} \sum_{j=0}^{\varkappa+1}(-1)^{j} \frac{B_{2 j}}{(2 j) !} \frac{B_{2 \varkappa+2-2 j}}{(2 \varkappa+2-2 j) !} x^{2 \varkappa+2-2 j} \\
& + \begin{cases}-\frac{1}{2} \zeta(2 \varkappa+1)\left\{1+(-1)^{\varkappa+1} x^{2 \varkappa}\right\} & \text { if } \varkappa \geq 1, \\
\frac{1}{2} \log x & \text { if } \varkappa=0,\end{cases}
\end{aligned}
$$

valid for Rex $>0$. (ii) The case $a=2 \varkappa$ is Guinand's formula, cf. ([29], Theorem 3) with

$$
P_{2 \varkappa}(x)=(2 \varkappa) ! \zeta(2 \varkappa+2)(2 \pi x)^{-2 \varkappa-1}+\frac{1}{2} \zeta(-2 \varkappa-1)(2 \pi x)
$$$$
+\frac{(-1)^{\varkappa}}{2}(2 \pi)^{-2 \varkappa}(2 \varkappa) ! \zeta(2 \varkappa+1), \quad \varkappa \geq 1 .
$$

(iii) The case $a=2 \varkappa+1$ reads

$$
I_{2 \varkappa+1}(x)=(-1)^{\varkappa+1}(2 \pi)^{-2 \varkappa-1} x^{-2 \varkappa-2} I_{2 \varkappa+1}\left(\frac{1}{x}\right)+P_{2 \varkappa+1}(x),
$$

where

$$
P_{2 \varkappa+1}(x)=(2 \varkappa+1) ! \zeta(2 \varkappa+2)(2 \pi x)^{-2 \varkappa-2}-\frac{1}{2} \zeta(-2 \varkappa-1)(\varkappa \geq 1) .
$$

These lead to (21), thence to the automorphy (27).

(iv) The special case of (50) with $x=1$, i.e., once differentiated form of Ramanujan's formula, yields Terras' formula [30, 31]:

$$
\zeta(3)=\frac{2}{45} \pi^{3}-4 \sum_{k=1}^{\infty} e^{-2 \pi k} \sigma_{-3}(k)\left(2 \pi^{2} k^{2}+\pi k+\frac{1}{2}\right) .
$$

Remark 2. The Mellin inversion with shifted argument (34) is an additive version of the "pseudomodular relation principle," which is a processed modular relation with the processing gamma factor $\Gamma(s+a)([11]$, p. 50$)$. In this special case, the Main Formula (38) is the manifestation of the statement of Razar that the differentiation of Lambert series essentially corresponds to the shift of the argument of the associated Dirichlet series.

Remark 3. It is shown ([11], pp. 82-84) that the special case $\varkappa=0, a=0$ of Theorem 2, the Ramanujan-Guinand formula, is nothing other than the automorphic property of the Dedekind eta-function, cf. Remark 1 above. This can be regarded as once differentiated form of Guinand's formula. We note that, in the case of $x=0$, the twice differentiated Guinand's formula, with a suitable modification, coincides with the automorphic property of the Eisenstein series $E_{2}$ : 


$$
E_{2}(\tau)+\frac{3}{\pi y}=1-24 \sum_{k=1}^{\infty} \frac{k q^{k}}{1-q^{k}}=\frac{12}{\pi i}(\log \eta(\tau))^{\prime} .
$$

Equation (51), once differentiated form of Guinand's formula $(=(2 n+1)$ times differentiated form of Ramanujan's formula=the negative case thereof) leads to (27), automorphy of $G_{2 \varkappa+2}$.

\section{Lipschitz Summation Formula}

Knopp-Robbins [7] in their Remark 1 state their view on the Lipschitz summation formula to the effect that it is conceptually simpler than Riemann's original method of using the theta series. However, at least the special case of the Lipschitz summation formula (Theorem 3) which is applied by Chapman to establish the limit relation has already been used extensively and can be readily deduced from the partial fraction expansion for the cotangent function. Since it is known that the partial fraction expansion is equivalent to the functional equation for the Riemann zeta-function, we may say that Chapman's result is a consequence of the functional equation. By Corollary 4 below, we shall show that the Lipschitz summation formula itself is equivalent to the functional equation, thereby enhancing the above statement. Pasles and Pribitkin [32] extend the Lipschitz summation formula to the two-variable case to which we hope to return elsewhere.

Theorem 3 (Lipschitz summation formula). For the complex variables $z=x+i y, x>0, s=\sigma+i t, \sigma>1$ and the real parameter $0<\alpha \leq 1$, we have the Lipschitz summation formula:

$$
\frac{(2 \pi)^{s}}{\Gamma(s)} \sum_{m=0}^{\infty}(m+\alpha)^{s-1} e^{-2 \pi z(m+\alpha)}=\sum_{n=-\infty}^{\infty} \frac{e^{2 \pi i n \alpha}}{(z+i n)^{s}}
$$

Under the condition $0<\alpha<1$, this formula holds in the wider half-plane $\sigma>0$.

In what follows, we shall generalize Theorem 3 in a wider framework, deducing Corollary 4 to Theorem 4 as a general modular relation.

Let

$$
\Phi(w, s, z)=\sum_{n=0}^{\infty} \frac{w^{n}}{(n+z)^{s}}
$$

be the Hurwitz-Lerch zeta-function, and let

$$
\phi(x, s, z)=\Phi\left(e^{2 \pi i n x}, s, x\right)=\sum_{n=0}^{\infty} \frac{e^{2 \pi i n x}}{(n+z)^{s}}
$$

denote the boundary function-the Lipschitz-Lerch transcendent (([9], pp. 59-62), ([2], pp. 128-131), [33]). This is in close correspondence with the case of $L_{s}(z)$ and $\ell_{s}(x)$ considered in [34]. As a consequence of the main formula in Appendix Section, we may deduce, from the reciprocal Hurwitz formula (64), a generalized Lipschitz summation formula (78) which is indeed a form of the functional equation.

Lemma 1. The Dirichlet series

$$
\begin{aligned}
\varphi(s) & =\frac{1}{\pi^{s / 2}} \ell_{s}(x)=\sum_{n=1}^{\infty} \frac{e^{2 \pi i x n}}{(\sqrt{\pi} n)^{s}}, \\
\psi_{1}(s) & =\frac{1}{2 \pi^{(s / 2)}} \zeta(s, x)+\frac{1}{2 \pi^{(s / 2)}} \zeta(s, 1-x) \\
& =\sum_{n=1}^{\infty}\left(\frac{(1 / 2)}{(\sqrt{\pi}(n+x-1))^{s}}+\frac{(1 / 2)}{(\sqrt{\pi}(n-x))^{s}}\right), \\
\psi_{2}(s) & =-\frac{i}{2 \pi^{(s / 2)}} \zeta(s, x)+\frac{i}{2 \pi^{(s / 2)}} \zeta(s, 1-x) \\
& =\sum_{n=1}^{\infty}\left(\frac{-(i / 2)}{(\sqrt{\pi}(n+x-1))^{s}}+\frac{(i / 2)}{(\sqrt{\pi}(n-x))^{s}}\right),
\end{aligned}
$$

satisfy the ramified functional equation, a special case of (A.2) with $r=1$

$$
\begin{aligned}
& \Gamma\left(\frac{s}{2}\right) \Gamma\left(\frac{1}{2}+\frac{s}{2}\right) \varphi(s) \\
& =\Gamma\left(\frac{1}{2}-\frac{s}{2}\right) \Gamma\left(\frac{1}{2}+\frac{s}{2}\right) \psi_{1}(1-s)+\Gamma\left(-\frac{s}{2}\right) \Gamma\left(1+\frac{s}{2}\right) \psi_{2}(1-s),
\end{aligned}
$$

or

$$
\begin{aligned}
& \chi(s) \\
& =\left\{\begin{array}{l}
\Gamma\left(s \mid-\left(0, \frac{1}{2}\right),\left(\frac{1}{2}, \frac{1}{2}\right) ; ;--\right) \varphi(s), \\
\Gamma\left(1-s \mid\left(0, \frac{1}{2}\right)\left(0, \frac{1}{2}\right) ; ;--\right) \psi_{1}(1-s)+\Gamma\left(1-s \mid\left(-\frac{1}{2}, \frac{1}{2}\right)\left(-\frac{1}{2}, \frac{1}{2}\right) ; ;--\right) \psi_{2}(1-s) .
\end{array}\right.
\end{aligned}
$$


Proof. From Euler's formula, we have

$$
\left\{\begin{array}{l}
e^{(s / 2) i(1-s)}=\sin \frac{s}{2} s+i \cos \frac{s}{2} s, \\
e^{-(s / 2) i(1-s)}=\sin \frac{s}{2} s-i \cos \frac{s}{2} s .
\end{array}\right.
$$

We transform the reciprocal Hurwitz formula $(0<x<1)$ :

$$
\begin{aligned}
\ell_{s}(x)= & \frac{\Gamma(1-s)}{(2 \pi)^{1-s}}\left(e^{\frac{1-s}{2} \pi i} \zeta(1-s, x)+e^{-((1-s) / 2) \pi i} \zeta(1-s, 1-x)\right) \\
& =f_{1}(s) f_{2}(s),
\end{aligned}
$$

say, where $f_{1}(s)=\left(\Gamma(1-s) /(2 \pi)^{1-s}\right)$ is the first factor of the right-hand side member and $f_{2}(s)$ is the second. Substituting (63), the second factor becomes

$$
\begin{aligned}
f_{2}(s)= & \sin \frac{\pi}{2} s(\zeta(1-s, x)+\zeta(1-s, 1-x)) \\
& +\cos \frac{\pi}{2} s(\zeta(1-s, x)-\zeta(1-s, 1-x)) .
\end{aligned}
$$

In view of this, we transform the first factor accordingly

$$
\begin{aligned}
f_{1}(s) & =\frac{\pi^{s-(3 / 2)}}{2} \frac{\Gamma((1-s) / 2)}{\Gamma(s / 2)} \frac{\pi}{\sin (\pi / 2) s} \\
\text { or } & =\frac{\pi^{s-(3 / 2)}}{2} \frac{\Gamma(1-(s / 2))}{\Gamma((1+s) / 2)} \frac{\pi}{\cos (\pi / 2) s} .
\end{aligned}
$$

Substituting (65) and (66), we transform (64) into

$$
\begin{aligned}
\ell_{s}(x)= & \frac{\pi^{s-(1 / 2)} \Gamma((1 / 2)-(s / 2))}{2 \Gamma((s / 2))}(\zeta(1-s, x)-\zeta(1-s, 1-x)) \\
& +i \frac{\pi^{s-(1 / 2)} \Gamma(1-(s / 2))}{2 \Gamma((s / 2)+(1 / 2))}(\zeta(1-s, x)-\zeta(1-s, 1-x)) \\
= & \frac{\pi^{-(s / 2)} \Gamma((1 / 2)-(s / 2))}{2 \Gamma(s / 2)} \psi_{1}(s)-\frac{\pi^{-(s / 2)} \Gamma(1-(s / 2))}{2 \Gamma((s / 2)+(1 / 2))} \psi_{2}(s),
\end{aligned}
$$

where $\psi_{i}^{\prime}$ 's are defined in (59) and (60). Clearing the denominators in (67),

$$
\begin{aligned}
\varphi(s) & =\Gamma\left(\frac{s}{2}\right) \Gamma\left(\frac{1}{2}+\frac{s}{2}\right) \frac{1}{\pi^{s / 2}} l_{s}(x) \\
& =\Gamma\left(\frac{1}{2}-\frac{s}{2}\right) \Gamma\left(\frac{1}{2}+\frac{s}{2}\right) \psi_{1}(s)-\Gamma\left(\frac{s}{2}\right) \Gamma\left(1-\frac{s}{2}\right) \psi_{2}(s) .
\end{aligned}
$$

It remains to note that the factor $-\Gamma(s / 2) \Gamma(1-(s / 2))$ of the second summand may be transformed into $\Gamma(-(s / 2)) \Gamma(1+(s / 2))$, which is indeed the case in view of the reciprocity formula.

By Lemma 1, we have the ramified functional equation. The only pole of $\chi(s)$ which is neither a pole of $\Gamma((1 / 2)+$ $(s / 2))$ nor of $\Gamma(1+(s / 2))$ is $s_{1}=0$. Thus, by main formula (A.13), we have the following modular relation. For the notation and formulas, we refer to ([11], pp. 49-59).

\section{Theorem 4}

$$
\begin{aligned}
& \frac{1}{\pi^{(s / 2)}} \sum_{n=1}^{\infty} \frac{e^{2 \pi i n x}}{n^{s}} H\left(z \sqrt{\pi} n \mid \Delta \oplus\left(\begin{array}{c}
- \\
\quad(s / 2),(1 / 2)),((1 / 2)+(s / 2),(1 / 2)) ;-
\end{array}\right)\right) \\
& =\frac{1}{2 \pi^{(1-s / 2)}} \sum_{n=1}^{\infty}\left\{\frac{1}{(n+x-1)^{1-s}} H\left(\frac{\sqrt{\pi}(n+x-1)}{z} \mid \Delta^{*} \oplus\left(\begin{array}{c}
((1-s / 2),(1 / 2)) ;- \\
((1-s / 2),(1 / 2)) ;-
\end{array}\right)\right)\right. \\
& \left.+\frac{1}{(n-x)^{1-s}} H\left(\frac{\sqrt{\pi}(n-x)}{z} \mid \Delta^{*} \oplus\left(\begin{array}{c}
((1-s / 2),(1 / 2)) ;- \\
((1-s / 2),(1 / 2)) ;-
\end{array}\right)\right)\right\} \\
& +\frac{1}{2 \pi^{(1-s) / 2}} \sum_{k=1}^{\infty}\left\{\frac{-i}{(n+x-1)^{1-s}} H\left(\frac{\sqrt{\pi}(n+x-1)}{z} \mid \Delta^{*} \oplus\left(\begin{array}{c}
(-(s / 2),(1 / 2)) ;- \\
(-(s / 2),(1 / 2)) ;-
\end{array}\right)\right)\right. \\
& \left.+\frac{i}{(n-x)^{1-s}} H\left(\frac{\sqrt{\pi}(n-x)}{z} \mid \Delta^{*} \oplus\left(\begin{array}{c}
(-(s / 2),(1 / 2)) ;- \\
(-(s / 2),(1 / 2)) ;-
\end{array}\right)\right)\right\}+\operatorname{Res}\left(\Gamma(w-s \mid \Delta) \chi(w) z^{s-w}, w=0\right) \\
& =\frac{1}{2 \pi^{(1-s) / 2}} \sum_{n=1}^{\infty}\left\{\frac { 1 } { ( n + x - 1 ) ^ { 1 - s } } \left\{H\left(\frac{\sqrt{\pi}(n+x-1)}{z} \mid \Delta^{*} \oplus\left(\begin{array}{c}
((1-s / 2),(1 / 2)) ;- \\
((1-s / 2),(1 / 2)) ;-
\end{array}\right)\right)\right.\right.
\end{aligned}
$$




$$
\begin{aligned}
& \left.-i H\left(\frac{\sqrt{\pi}(k+a-1)}{z} \mid \Delta^{*} \oplus\left(\begin{array}{r}
(-(s / 2),(1 / 2)) ;- \\
(-(s / 2),(1 / 2)) ;-
\end{array}\right)\right)\right\} \\
& \left.+\frac{1}{(n-x)^{1-s}}\left\{H\left(\frac{\sqrt{\pi}(n-x)}{z} \mid \Delta^{*} \oplus\left(\begin{array}{c}
((1-s / 2),(1 / 2)) ;- \\
((1-s / 2),(1 / 2)) ;-
\end{array}\right)\right)+i H\left(\frac{\sqrt{\pi}(n-x)}{z} \mid \Delta^{*} \oplus\left(\begin{array}{c}
(-(s / 2),(1 / 2)) ;- \\
(-(s / 2),(1 / 2)) ;-
\end{array}\right)\right)\right\}\right\} \\
& +\operatorname{Res}\left(\Gamma(w-s \mid \Delta) \chi(w) z^{s-w}, w=0\right) .
\end{aligned}
$$

Corollary 2. If $\Delta=\left(\begin{array}{cc}(1,1) & ;- \\ - & ;-\end{array}\right)$, we have $\Delta^{*}=\left(\begin{array}{cc}- & ;- \\ (0,1) & ;-\end{array}\right)$, and so Theorem 4 amounts to

$$
\begin{aligned}
& \frac{1}{\pi^{(s / 2)}} \sum_{n=1}^{\infty} \frac{e^{2 \pi i n a}}{n^{s}} H_{2,1}^{1,2}\left(\begin{array}{l|l}
z \sqrt{\pi} n & (1,1) \\
\left(\frac{s}{2}, \frac{1}{2}\right),\left(\frac{1}{2}+\frac{s}{2}, \frac{1}{2}\right)
\end{array}\right) \\
& =\frac{1}{2 \pi^{(1-s / 2)}} \sum_{n=1}^{\infty}\left\{\frac { 1 } { ( n + a - 1 ) ^ { 1 - s } } \left\{H_{1,2}^{2,1}\left(\frac{\sqrt{\pi}(n+a-1)}{z} \mid \begin{array}{c}
\left(\frac{1-s}{2}, \frac{1}{2}\right) \\
(0,1),\left(\frac{1-s}{2}, \frac{1}{2}\right)
\end{array}\right)\right.\right. \\
& \left.-i H_{1,2}^{2,1}\left(\frac{\sqrt{\pi}(n+a-1)}{z} \mid \begin{array}{c}
\left(-\frac{s}{2}, \frac{1}{2}\right) \\
(0,1),\left(-\frac{s}{2}, \frac{1}{2}\right)
\end{array}\right)\right\} \\
& +\frac{1}{(n-a)^{1-s}}\left\{\begin{array}{l|l}
H_{1,2}^{2,1}\left(\frac{\sqrt{\pi}(n-a)}{z}\right. & \left(\frac{1-s}{2}, \frac{1}{2}\right) \\
& (0,1),\left(\frac{1-s}{2}, \frac{1}{2}\right)
\end{array}\right) \\
& \left.\left.+i H_{1,2}^{2,1}\left(\begin{array}{c|c}
\frac{\sqrt{\pi}(n-a)}{z} & \left(-\frac{s}{2}, \frac{1}{2}\right) \\
(0,1),\left(-\frac{s}{2}, \frac{1}{2}\right)
\end{array}\right)\right\}\right\} \\
& +\operatorname{Res}\left(\Gamma(s-w) \chi(w) z^{s-w}, w=0\right) .
\end{aligned}
$$

Corollary 3 (Ewald expansion).

$$
\begin{aligned}
\phi(x, s, z)= & \sum_{n=1}^{\infty} \frac{e^{2 \pi i n x}}{(n+z)^{s}}+\frac{1}{z^{s}} \\
= & \sum_{n=1}^{\infty}\left(\frac{e^{-2 \pi(n+x-1) z i}}{\left(2 \pi(n+x-1) e^{-(\pi i / 2)}\right)^{1-s}} \Gamma(1-s,-2 \pi(n+x-1) z i)+\frac{e^{2 \pi(n-x) z i}}{\left(2 \pi(n-x) e^{\pi i / 2}\right)^{1-s}} \Gamma(1-s, 2 \pi(n-x) z i)\right) \\
& +\frac{1}{2 z^{s}},
\end{aligned}
$$


where $\Gamma(s, c)$ is the incomplete gamma function defined as follows:

$$
\Gamma(s, c)=\Gamma(s)-c^{s} \int_{0}^{1} e^{-c u} u^{s-1} \mathrm{~d} u
$$

Proof. We transform $H$-functions in Corollary 2 in a concrete form. The procedure is the same for the three $H$-functions, i.e., duplication formula, $H \longrightarrow G$ formula, and the explicit formula for the $G$-function, and we use the known results on them freely, cf. [11, 35], etc. We have

$$
\begin{aligned}
H_{2,1}^{1,2}\left(z \mid \begin{array}{l}
(1,1) \\
\left(\frac{s}{2}, \frac{1}{2}\right),\left(\frac{1}{2}+(s / 2), \frac{1}{2}\right)
\end{array}\right) & =2^{1-s} \sqrt{\pi} G_{1,1}^{1,1}\left(2 z \mid \begin{array}{l}
1 \\
s
\end{array}\right) \\
& =2^{1-s} \sqrt{\pi} \Gamma(s) \frac{(2 z)^{s}}{(1+2 z)^{s}},
\end{aligned}
$$

$$
\begin{aligned}
& H_{1,2}^{2,1}\left(\begin{array}{l}
\left(\begin{array}{l}
\left(\frac{1}{2}-\frac{s}{2}, \frac{1}{2}\right) \\
(0,1),\left(\frac{1}{2}-\frac{s}{2}, \frac{1}{2}\right)
\end{array}\right) \\
=\frac{1}{2 \sqrt{\pi}} H_{1,3}^{3,1}\left(\begin{array}{l|l}
\left.\frac{z}{2}-\frac{s}{2}, \frac{1}{2}\right) \\
\left(0, \frac{1}{2}\right)
\end{array}\right),\left(\frac{1}{2}, \frac{1}{2}\right),\left(\frac{1}{2}-\frac{s}{2}, \frac{1}{2}\right)
\end{array}\right) \\
& =\frac{1}{\sqrt{\pi}} G_{1,3}^{3,1}\left(\begin{array}{l}
\frac{1-s}{2} \\
z^{2} \\
0, \frac{1}{2}, \frac{1-s}{2}
\end{array}\right) \\
& =\Gamma(s)\left(e^{((1-s) / 2) \pi i-z i} \Gamma(1-s,-z i)+e^{-((1-s) / 2) \pi i+z i} \Gamma(1-s, z i)\right),
\end{aligned}
$$

and similarly to (74)

$$
\begin{aligned}
& H_{1,2}^{2,1}\left(\begin{array}{l}
z\left(-\frac{s}{2}, \frac{1}{2}\right) \\
(0,1),\left(-\frac{s}{2}, \frac{1}{2}\right)
\end{array}\right) \\
& =\Gamma(s+1)\left(e^{-(s / 2) \pi i-z i} \Gamma(-s,-z i)+e^{(s / 2) \pi i+z i} \Gamma(-s, z i)\right) \\
& =\Gamma(s)\left(i e^{(1-s / 2) \pi i-z i} \Gamma(1-s,-z i)-i e^{-(1-s / 2) \pi i+z i} \Gamma(1-s, z i)+\frac{2}{z^{s}}\right) .
\end{aligned}
$$

$$
\begin{aligned}
& \frac{1}{(2 \sqrt{\pi})^{s-1}} \sum_{n=1}^{\infty} \frac{e^{2 \pi i n x}}{(n+\xi)^{s}} \\
& =\frac{1}{\pi^{((1-s) / 2)}} \sum_{n=1}^{\infty}\left\{\frac{e^{((1-s) / 2) \pi i} e^{-2 \pi(n+x-1) \xi i}}{(n+x-1)^{1-s}} \Gamma\right. \\
& \cdot(1-s,-2 \pi(n+x-1) \xi i) \\
& +\frac{e^{-((1-s) / 2) \pi i} e^{2 \pi(n-x) \xi i}}{(n-x)^{1-s}} \Gamma(1-s, 2 \pi(n-x) \xi i) \\
& \left.\quad-\frac{i}{(2 \pi \xi)^{s}(n+x-1)}+\frac{i}{(2 \pi \xi)^{s}(n-x)}\right\} \\
& +\frac{1}{(2 \sqrt{\pi})^{s-1} \xi^{s}} \ell_{0}(x),
\end{aligned}
$$

where $\xi=(1 / 2 \sqrt{\pi} z)$. Since

$$
\ell_{0}(x)=-\frac{1}{2}+\frac{i}{2 \pi}\left(\frac{1}{x}+\sum_{n=1}^{\infty}\left(\frac{1}{x+n}+\frac{1}{x-n}\right)\right)
$$

equation (76) leads to the incomplete gamma series (71) for (57).

We are now in a position to transform (71) into the functional equation.

Corollary 4 (General Lipschitz summation formula)

$$
\begin{aligned}
\phi(x, s, z) & \\
= & \frac{\Gamma(1-s)}{(2 \pi)^{1-s}}\left(e^{(1-s / 2) \pi i-2 \pi x z i} \phi(-z, 1-s, x)\right. \\
& \left.+e^{-(1-s / 2) \pi i+2 \pi(1-x) z i} \phi(z, 1-s, 1-x)\right),
\end{aligned}
$$

where $0<z, x<1$, and $t=-z(u-1)$.

Proof. By (72), we have the left-hand side of (71) is equal to

$$
\begin{aligned}
= & \Gamma(1-s) \sum_{k=1}^{\infty}\left(\frac{e^{-2 \pi(n+x-1) z i}}{\left(2 \pi(n+x-1) e^{-(\pi i / 2)}\right)^{1-s}}+\frac{e^{2 \pi(n-x) z i}}{\left(2 \pi(n-x) e^{(\pi i / 2)}\right)^{1-s}}\right) \\
& -\frac{1}{z^{s-1}} \sum_{k=1}^{\infty} \int_{0}^{1}\left(e^{2 \pi(n+x-1) z i(u-1)}+e^{-2 \pi(n-x) z i(u-1)}\right) u^{-s} \mathrm{~d} u+\frac{1}{2 z^{s}} \\
= & \frac{\Gamma(1-s)}{(2 \pi)^{1-s}}\left(e^{(1-s) / 2 \pi i-2 \pi x z i} \phi(-z, 1-s, x)\right. \\
& \left.+e^{-(1-s / 2) \pi i+2 \pi(1-z) z i} \phi(z, 1-s, 1-x)\right) \\
& -\frac{1}{z^{s}} \sum_{k \in \mathbb{Z}} \int_{0}^{z} e^{2 \pi(n-x) t i}\left(1-\frac{t}{z}\right)^{-s} \mathrm{~d} t+\frac{1}{2 z^{s}} .
\end{aligned}
$$

Hence, (71) amounts to 
Equation (78) is sometimes referred to as the Lipschitz summation formula ([36-38] etc.).

The character analogue of the Lipschitz summation formula is known ([2], pp. 128-131), and so we may naturally treat a more general case which will be conducted elsewhere.

We briefly state the Lipschitz summation formula for quadratic fields which is contained in ([11], Chapter 4). This is an elucidation of Koshlyakov's results [39-42]. Let $\Omega$ be a quadratic field whose degree $x=r_{1}+r_{2}=2$ with its discriminant $\Delta$. Let

$$
A=\frac{2^{r_{2}} \pi^{\varkappa / 2}}{\sqrt{|\Delta|}},
$$

and let $r=r_{1}+r_{2}-1$ denote the rank of the unit group. Let $\zeta_{\Omega}(s)$ be the Dedekind zeta-function of $\Omega$ with the coefficients $\alpha_{k}=\beta_{k}=\alpha(k)$, with $\alpha(k)$ indicating the number of ideals of norm $k$, where $\lambda_{k}=A k, \mu_{k}=A k, r=1$, and

$$
\rho=\frac{2^{r+1} \pi^{r_{2}} R h}{w \sqrt{|\Delta|}}=-\frac{2^{r+1} \pi^{r_{2}} \zeta_{\Omega}^{(r)}(s)}{\sqrt{|\Delta|}} .
$$

The functional equation reads

$$
\Gamma^{r_{1}}\left(\frac{1}{2} s\right) \Gamma^{r_{2}}(s) \varphi(s)=\Gamma^{r_{1}}\left(\frac{1}{2}-\frac{1}{2} s\right) \Gamma^{r_{2}}(1-s) \varphi(1-s),
$$

where

$$
\varphi(s)=A^{-s} \zeta_{\Omega}(s)=\sum_{n=1}^{\infty} \frac{\alpha(n)}{(A n)^{s}} .
$$

In conformity with Koshlyakov ([40], p. 241) (cf. (83)), we introduce the perturbed Dedekind zeta-function:

$$
\zeta_{\Omega}(s, z)=-\frac{2 \zeta_{\Omega}(0)}{w}+\sum_{n=1}^{\infty} \frac{\alpha(n)}{(n+z)^{s}} .
$$

Then the Fourier-Bessel expansion gives the Lipschitz summation formula for an imaginary quadratic field ([11], (4.42)):

$$
\begin{aligned}
\sum_{n=-\infty}^{\infty} \frac{\alpha(n)}{(z+i n)^{s}}= & e^{(\pi i / 2) s} \zeta_{\Omega}(s, i z)+e^{-(\pi i / 2 s)} \zeta_{\Omega}(s,-i z) \\
= & A^{s} \frac{z^{(1 / 2)(1-s)}}{\Gamma(s)} \sum_{n=1}^{\infty} \frac{\alpha(n)}{n^{(1 / 2)(1-s)}}\left(\varepsilon^{s+1} K_{1-s}(2 A \varepsilon \sqrt{n z})\right. \\
& \left.+\bar{\varepsilon}^{s+1} K_{1-s}(2 A \bar{\varepsilon} \sqrt{n z})\right) \\
& -\frac{\zeta_{\Omega}(0)}{z^{s}}
\end{aligned}
$$

which is the corrected form of (23.15) in [40], where $\varepsilon=$ $e^{(\pi / 4) i}$ and the prime on the summation sign means that the term with $n=0$ is excluded.

Theorem 5 (Theorem 4.6 in [11]). The generating Dirichlet series for the Wigert-Bellman divisor problem, cf. (29) above,

$$
\begin{aligned}
\zeta_{\Omega}(s, z)= & \frac{z^{-s}}{\Gamma(s)} \sum_{n=1}^{\infty} \frac{\alpha(n)}{n} \frac{1}{2 \pi i} \\
& \cdot \int_{(c)} \tan \frac{\pi}{2} w \Gamma(1-w) \Gamma(s-w)\left(\frac{1}{n z}\right)^{-w} \mathrm{~d} w
\end{aligned}
$$

amounts to the Lipschitz summation formula

$$
\begin{aligned}
\frac{1}{2} & \sum_{n=-\infty}^{\infty} \frac{\alpha(n)}{(z+i n)^{s}}=\frac{1}{2} e^{(\pi i / 2) s} \zeta_{\Omega}(s, i z)+\frac{1}{2} e^{-(\pi i / 2) s} \zeta_{\Omega}(s,-i z) \\
& =A^{s} \frac{z^{(1 / 2)(1-s)}}{\Gamma(s)} \sum_{n=1}^{\infty} \frac{\alpha(n)}{n^{(1 / 2)(1-s)}}\left(\frac{\varepsilon^{s+1}}{i} K_{1-s}(2 A \varepsilon \sqrt{n z})\right. \\
& \left.-\frac{\bar{\varepsilon}^{s+1}}{i} K_{1-s}(2 A \bar{\varepsilon} \sqrt{n z})\right) .
\end{aligned}
$$

This is the corrected form of (23.16) in [40].

\section{Ramified Functional Equations}

There are some instances of the ramified functional equations in literature.

In [43-45], they are stated in the case of zeta-functions with periodic coefficients which satisfy the ramified functional equations as a result of representations in bases consisting of the Hurwirz and Lerch zeta-functions [18]: suppose $f(n)$ be a periodic function with period $M$,

$$
L(s, f)=\sum_{n=1}^{\infty} \frac{f(n)}{n^{s}},
$$

be the associated Dirichlet series absolutely convergent $\sigma=$ Res $>1$ and that

$$
\begin{aligned}
& f_{\text {odd }}=\frac{1}{2}(f(n \bmod M)-f(-n \bmod M)), \\
& f_{\text {even }}=\frac{1}{2}(f(n \bmod M)+f(-n \bmod M)),
\end{aligned}
$$

be odd, resp. even part of $f: f=f_{\text {even }}+f_{\text {odd }}$. Then

$$
\begin{aligned}
L(1-s, f)= & \left(\frac{\pi}{M}\right)^{(1 / 2)-s}\left(\frac{\Gamma(s / 2)}{\Gamma(1-s / 2)} L\left(s, \widehat{f}_{\text {even }}\right)\right. \\
& \left.+\frac{\Gamma((1+s) / 2)}{\Gamma(1-(s / 2))} L\left(s, \widehat{f}_{\text {odd }}\right)\right),
\end{aligned}
$$

which amounts to (61) on clearing the denominators and multiplying by $(\pi / M)^{(s-1) / 2}$. Wang and Banerjee [46] treat the product of Hurwitz zeta-functions which satisfy a ramified functional equation as a result of the Hurwitz formula:

$$
\zeta(1-s, x)=\frac{\Gamma(s)}{(2 \pi)^{s}}\left(e^{-(\pi i s / 2)} \ell_{s}(x)+e^{(\pi i s / 2)} \ell_{s}(1-x)\right),
$$


whose reciprocal (64) has been used and proved to be a ramified functional equation in the proof of Lemma 1 above. More general case is that of the Hurwitz-Lerch zeta-function described in [35] (pp. 27-31) and [33] (pp. 121-126, 339-341). Another class of zeta-functions that satisfy a ramified functional equations is that of Barnes multiple zetafunctions cf. [33] (pp. 77-88) and [47]. For their rich applications [48] and references therein. Hardy and Littlewood $[49,50]$ use the Barnes double zeta-function.

In another context, Estermann [51] and others and in Prehomogeneous Vector Space (PHV) theory [52].

\section{Appendix}

\section{The Main Formula}

In this section, we state a special case $(H=1)$ of the Main Formula in [11] (Section 4.4, pp. 115-122) first proved in [53] which was used in the proof of Theorem 4 . We have two sets of Dirichlet series $\{\phi(s)\}$ and $\left\{\psi_{i}(s)\right\}, 1 \leq i \leq I$ that satisfy the generalized functional equation (A.2) in the following sense.

With increasing sequences $\left\{\lambda_{k}\right\}_{k=1}^{\infty},\left\{\mu_{k}^{(i)}\right\}_{k=1}^{\infty}(1 \leq i \leq I)$ and complex sequences $\left\{\alpha_{k}\right\}_{k=1}^{\infty},\left\{\beta_{k}^{(i)}\right\}_{k=1}^{\infty}(1 \leq i \leq I)$, we form the Dirichlet series:

$$
\begin{gathered}
\varphi(s)=\sum_{k=1}^{\infty} \frac{\alpha_{k}}{\lambda_{k}^{s}} \\
\psi_{i}(s)=\sum_{k=1}^{\infty} \frac{\beta_{k}^{(i)}}{\mu_{k}^{(i) s}},
\end{gathered}
$$

$$
1 \leq i \leq I
$$

which we suppose have finite abscissa of absolute convergence $\sigma_{\varphi}, \sigma_{\psi_{i}}(1 \leq i \leq I)$, respectively.

We assume the existence of the meromorphic function $\chi$, which satisfies, for a real number $r$, the functional equation:

$$
=\left\{\begin{array}{l}
\frac{\prod_{j=1}^{M} \Gamma\left(d_{j}+D_{j} s\right) \prod_{j=1}^{N} \Gamma\left(c_{j}-C_{j} s\right)}{\prod_{j=N+1}^{P} \Gamma\left(c_{j}+C_{j} s\right) \prod_{j=M+1}^{Q} \Gamma\left(d_{j}-D_{j} s\right)} \varphi_{h}(s), \quad R e(s)>\sigma_{\varphi}, \\
\sum_{i=1}^{I} \prod_{j=1}^{(i)} \Gamma\left(e_{j}^{(i)}+E_{j}^{(i)}(r-s)\right) \frac{\prod_{j=1}^{\tilde{M}^{(i)}} \Gamma\left(f_{j}^{(i)}-F_{j}^{(i)}(r-s)\right)}{\prod_{j=\tilde{M}^{(i)}+1}^{\tilde{Q}^{(i)}} \Gamma\left(-b \pm \sqrt{b^{2}-4 a c} / 2 a f_{j}^{(i)}+F_{j}^{(i)}(r-s)\right) \prod_{j=\tilde{N}^{(i)}+1}^{\tilde{P}^{(i)}} \Gamma\left(e_{j}^{(i)}-E_{j}^{(i)}(r-s)\right) \psi_{i}(r-s), \operatorname{Re}(s)<\min _{1 \leq i \leq I}\left(r-\sigma_{\psi_{i}}\right),\left(C_{j}, D_{j}, E_{j}^{(i)}, F_{j}^{(i)}>0\right) .}
\end{array}\right.
$$

We assume further that only finitely many of the poles $s_{k}(1 \leq k \leq L)$ of $\chi(s)$ are neither a pole of

$$
\frac{\prod_{j=1}^{N} \Gamma\left(c_{j}-C_{j} s\right)}{\prod_{j=N+1}^{P} \Gamma\left(c_{j}+C_{j} s\right) \prod_{j=M+1}^{Q} \Gamma\left(d_{j}-D_{j} s\right)},
$$

$$
\frac{\prod_{j=1}^{\tilde{M}^{(i)}} \Gamma\left(f_{j}^{(i)}-F_{j}^{(i)} r+F_{j}^{(i)} s\right)}{\prod_{j=\widetilde{N}^{(i)}+1}^{\widetilde{P}^{(i)}} \Gamma\left(e_{j}^{(i)}-E_{j}^{(i)} r+E_{j}^{(i)} s\right) \prod_{j=\widetilde{M}^{(i)}+1}^{(i)} \Gamma\left(f_{j}^{(i)}+F_{j}^{(i)} r-F_{j}^{(i)} s\right)} .
$$

We introduce the processing gamma factor:

nor a pole of

$$
\Gamma(w \mid \Delta)=\frac{\prod_{j=1}^{m} \Gamma\left(b_{j}+B_{j} w\right) \prod_{j=1}^{n} \Gamma\left(a_{j}-A_{j} w\right)}{\prod_{j=n+1}^{p} \Gamma\left(a_{j}+A_{j} w\right) \prod_{j=m+1}^{q} \Gamma\left(b_{j}-B_{j} w\right)}, \quad\left(A_{j}, B_{j}>0\right)
$$

and suppose that for any real numbers $u_{1}, u_{2} \quad\left(u_{1}<u_{2}\right)$,

$$
\lim _{|v| \longrightarrow \infty} \Gamma(u+i v-s \mid \Delta) \chi(u+i v)=0,
$$

uniformly in $u_{1} \leq u \leq u_{2}$.

We choose $L_{1}(s)$ so that the poles of

$$
\frac{\prod_{j=1}^{n} \Gamma\left(a_{j}+A_{j} s-A_{j} w\right) \prod_{j=1}^{N} \Gamma\left(c_{j}-C_{j} w\right)}{\prod_{j=n+1}^{p} \Gamma\left(a_{j}-A_{j} s+A_{j} w\right) \prod_{j=N+1}^{P} \Gamma\left(c_{j}+C_{j} w\right)} \times \frac{1}{\prod_{j=m+1}^{q} \Gamma\left(b_{j}+B_{j} s-B_{j} w\right) \prod_{j=M+1}^{Q} \Gamma\left(d_{j}-D_{j} w\right)},
$$


lie on the right of $L_{1}(s)$, and those of

$$
\frac{\prod_{j=1}^{m} \Gamma\left(b_{j}-B_{j} s+B_{j} w\right) \prod_{j=1}^{M} \Gamma\left(d_{j}+D_{j} w\right)}{\prod_{j=m+1}^{q} \Gamma\left(b_{j}+B_{j} s-B_{j} w\right) \prod_{j=M+1}^{Q} \Gamma\left(d_{j}-D_{j} w\right)} \times \frac{1}{\prod_{j=n+1}^{p} \Gamma\left(a_{j}-A_{j} s+A_{j} w\right) \prod_{j=N+1}^{P} \Gamma\left(c_{j}+C_{j} w\right)},
$$

lie on the left of $L_{1}(s)$, and choose $L_{2}(s)$ so that the poles of

$$
\frac{\prod_{j=1}^{m} \Gamma\left(b_{j}-B_{j} s+B_{j} w\right) \prod_{j=1}^{\tilde{M}^{(i)}} \Gamma\left(f_{j}^{(i)}-F_{j}^{(i)} r+F_{j}^{(i)} w\right)}{\prod_{j=m+1}^{q} \Gamma\left(b_{j}+B_{j} s-B_{j} w\right) \prod_{j=\tilde{M}^{(i)}+1}^{\widetilde{Q}^{(i)}} \Gamma\left(f_{j}^{(i)}+F_{j}^{(i)} r-F_{j}^{(i)} w\right)} \times \frac{1}{\prod_{j=n+1}^{p} \Gamma\left(a_{j}-A_{j} s+A_{j} w\right) \prod_{j=\tilde{N}^{(i)}+1}^{\tilde{P}^{(i)}} \Gamma\left(e_{j}^{(i)}-E_{j}^{(i)} r+E_{j}^{(i)} w\right)},
$$

lie on the left of $L_{2}(s)$, and those of

$$
\frac{\prod_{j=1}^{n} \Gamma\left(a_{j}+A_{j} s-A_{j} w\right) \prod_{j=1}^{\tilde{N}(i)} \Gamma\left(e_{j}^{(i)}+E_{j}^{(i)} r-E_{j}^{(i)} w\right)}{\prod_{j=n+1}^{p} \Gamma\left(a_{j}-A_{j} s+A_{j} w\right) \prod_{j=\tilde{N}^{(i)}+1}^{\widetilde{P}^{(i)}} \Gamma\left(e_{j}^{(i)}-E_{j}^{(i)} r+E_{j}^{(i)} w\right)} \times \frac{1}{\prod_{j=m+1}^{q} \Gamma\left(b_{j}+B_{j} s-B_{j} w\right) \prod_{j=\tilde{M}_{+1}^{(i)}}^{\widetilde{Q}^{(i)}} \Gamma\left(f_{j}^{(i)}+F_{j}^{(i)} r-F_{j}^{(i)} w\right)},
$$

lie on the right of $L_{2}(s)$. Further, they squeeze a compact set $S$ such that $s_{k} \in S(1 \leq k \leq L)$. Under these conditions, we define the $\chi$-function, key-function, $X(z, s \mid \Delta)$ by

$$
X(z, s \mid \Delta)=\frac{1}{2 \pi i} \int_{L_{1}(s)} \Gamma(w-s \mid \Delta) \chi(w) z^{-w} \mathrm{~d} w,
$$

where $\Gamma(s \mid \Delta)$ is the processing gamma factor (A.5).

Then, we have the following modular relation, equivalent to the functional equation (A.2):

The Main Formula $H$ :

$$
\begin{aligned}
& X(z, s \mid \Delta)=
\end{aligned}
$$

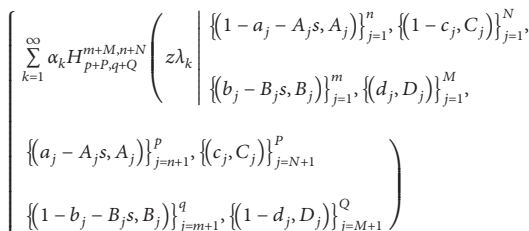

$$
\begin{aligned}
& \text { if } L_{1}(s) \text { can be taken to the right of } \sigma_{\varphi}
\end{aligned}
$$

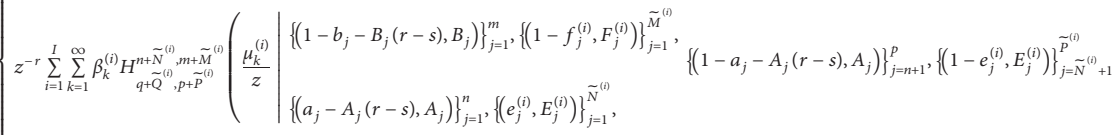

$$
\begin{aligned}
& \left\{\left(b_{j}-B_{j}(r-s), B_{j}\right)\right\}_{j=m+1}^{q},\left\{\left(f_{j}^{(i)}, F_{j}^{(i)}\right)\right\}_{j=\tilde{M}^{(i)}+1}^{\tilde{Q}^{(i)}} \\
& \left.\left\{\left(1-a_{j}-A_{j}(r-s), A_{j}\right)\right\}_{j=n+1}^{p},\left\{\left(1-e_{j}^{(i)}, E_{j}^{(i)}\right)\right\}_{j=\tilde{N}^{(i)}+1}^{\widetilde{P}^{(i)}}\right\} \\
& +\sum_{k=1}^{L} \operatorname{Res}\left(\Gamma(w-s \mid \Delta) \chi(w) z^{-w}, w=s_{k}\right) \\
& \text { if } L_{2}(s) \text { can be taken to the left of } \min _{1 \leq i \leq I}\left(r-\sigma_{\psi_{i}}\right) \text {, }
\end{aligned}
$$




$$
\begin{aligned}
& z^{s} \mathrm{X}(z, s \mid \Delta)=
\end{aligned}
$$

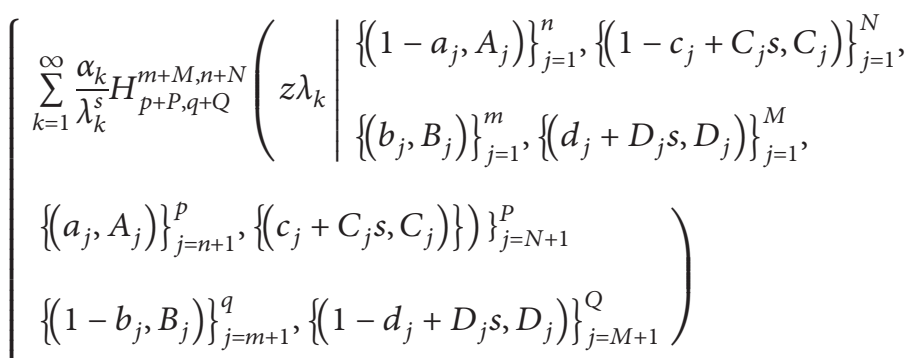

$$
\begin{aligned}
& \text { if } L_{1}(s) \text { can be taken to the right of } \sigma_{\varphi} \\
& \left\{\sum _ { i = 1 } ^ { I } \sum _ { k = 1 } ^ { \infty } \frac { \beta _ { k } ^ { ( i ) } } { \mu _ { k } ^ { ( i ) r - s } } H _ { \substack { n + \tilde { N } ^ { ( i ) } , { } ^ { ( i ) } , { } ^ { ( i ) } \tilde { P } ^ { ( i ) } } } ^ { \tilde { \sim } ^ { ( i ) } } \left(\frac{\mu_{k}^{(i)}}{z} \mid \begin{array}{l}
\left\{\left(1-b_{j}, B_{j}\right)\right\}_{j=1}^{m},\left\{\left(1-f_{j}^{(i)}+F_{j}^{(i)}(r-s), F_{j}^{(i)}\right)\right\}_{j=1}^{\tilde{M}^{(i)}}, \\
\left\{\left(a_{j}, A_{j}\right)\right\}_{j=1}^{n},\left\{\left(e_{j}^{(i)}+E_{j}^{(i)}(r-s), E_{j}^{(i)}\right)\right\}_{j=1}^{\tilde{N}^{(i)}},
\end{array},\right.\right. \\
& \left.\begin{array}{l}
\left\{\left(b_{j}, B_{j}\right)\right\}_{j=m+1}^{q},\left\{\left(f_{j}^{(i)}+F_{j}^{(i)}(r-s), F_{j}^{(i)}\right)\right\}_{j=\tilde{M}^{(i)}+1}^{\tilde{Q}^{(i)}} \\
\left\{\left(1-a_{j}, A_{j}\right)\right\}_{j=n+1}^{p},\left\{\left(1-e_{j}^{(i)}+E_{j}^{(i)}(r-s), E_{j}^{(i)}\right)\right\}_{j=\widetilde{N}^{(i)}+1}^{\widetilde{P}^{(i)}}
\end{array}\right) \\
& +\sum_{k=1}^{L} \operatorname{Res}\left(\Gamma(w-s \mid \Delta) \chi(w) z^{s-w}, w=s_{k}\right) \\
& \text { if } L_{2}(s) \text { can be taken to the left of } \min _{1 \leq i \leq I}\left(r-\sigma_{\psi_{i}}\right) \text {. }
\end{aligned}
$$

\section{Data Availability}

No data were used to support this study.

\section{Disclosure}

This study is dedicated to Professor Dr. Chaohua Jia on his sixtieth birthday with great respect and friendship.

\section{Conflicts of Interest}

The authors declare that they have no conflicts of interest.

\section{Acknowledgments}

The authors are grateful to Prof. Shigeru Kanemitsu and Dr. B.-W. Li for their kind help in this paper. The research of the third author is supported by the UGC-BSR Research StartUp-Grant (F.30-404/2017(BSR)).

\section{References}

[1] H. L. Li, J. Ma, and W. P. Zhang, "On some diophantine fourier series," Acta Mathematica Sinica, English Series, vol. 26, no. 6, pp. 1125-1132, 2010.

[2] F.-H. Li and S. Kanemitsu, "Around boundary functions of the right half-plane and the unit disc," Special Functions and Analysis of Differential Equations, to Appear, Chapman and Hall/CRC, London, UK, 2020.

[3] A. Wintner, "On Riemann's fragment concerning elliptic modular functions," American Journal of Mathematics, vol. 63, no. 3, pp. 628-634, 1941.

[4] N. Kurokawa, "Limit values of Eisenstein series and multiple cotangent functions," Journal of Number Theory, vol. 128, pp. 1776-1784, 2008.

[5] S.-y. Koyama and N. Kurokawa, "Multiple Eisenstein series and multiple cotangent functions," Journal of Number Theory, vol. 128, no. 6, pp. 1769-1774, 2008.

[6] R. Chapman, "Limit formulas for non-modular Eisenstein series," Journal of Combinatorics and Number Theory, vol. 1, no. 2, pp. 127-132, 2009.

[7] M. Knopp and S. Robins, "Easy proof of Riemann's functional equation for $\zeta(s)$ and of Lipschitz summation," Proceedings of the American Mathematical Society, vol. 129, no. 7, pp. 1915-1923, 2001.

[8] H. M. Stark, "Dirichlet's class-number formula revisited," A Tribute to Emil Grosswald: Number Theory and Related Analysis, vol. 143, pp. 571-577, 1993.

[9] K. Chakraborty, S. Kanemitsu, and H. Tsukada, Vistas of Special Functions II, World Scientific, Singapore, 2009.

[10] M. R. Murty and K. Sinha, "Multiple Hurwitz zeta functions," Proceedings of Symposia in Pure Mathematics, vol. 75, pp. 135-156, 2006. 
[11] S. Kanemitsu and H. Tsukada, Contributions to the Theory of Zeta-Functions: The Modular Relation Supremacy, World Scientific, Singapore, 2015.

[12] A. Weil, "Dirichlet series and automorphic forms," in Lecture Notes in Mathematics, Vol. 189, Springer, Berlin, Germany, 1971.

[13] M. Knopp, Hamburger's Theorem on $\zeta(s)$ and the Abundance Principle for Dirichlet Series with Functional Equations, Number Theory, R. P. Bambah, Ed., pp. 201-216, Hindustan Book Agency, New Delhi, India, 2000.

[14] J. H. Bruinier and J. Funke, "On two geometric theta lifts," Duke Mathematical Journal, vol. 125, no. 1, pp. 45-90, 2004.

[15] R. Bellman, "Wigert's approximate functional equation and the Riemann zeta-function," Duke Mathematical Journal, vol. 16, no. 4, pp. 547-552, 1949.

[16] S. Kanemitsu and H. Tsukada, Vistas of Special Functions, World Scientific, Singapore, 2007.

[17] F.-H. Li, N.-L. Wang, and S. Kanemitsu, Number Theory and its Applications, World Scientific, Singapore, 2012.

[18] X.-H. Wang and S. Kanemitsu, "On the product of zetafunctions, to appear," 2021.

[19] S. Bochner, "Some properties of modular relations," Annals of Mathematics, vol. 53, no. 2, pp. 332-363, 1951.

[20] J.-P. Serre, A Course in Arithmetic, Springer, Berlin, Germany, 1973.

[21] B. C. Berndt and A. Straub, Ramanujan's Formula for $\zeta(2 n+1)$, Exploring the Riemann Zeta Function, H. Montgomery, A. Nikeghbali, and M. Rassias, Eds., Springer, Berlin, Germany, 2017.

[22] A. Weil, "Sur une formule classique," Journal of the Mathematical Society of Japan, vol. 20, pp. 400-402, 1968.

[23] S. Chowla, "Remarks on class invariants and related topics," in Seminar on Complex Multiplication Lecture Notes in Mathematics, Springer, Berlin, Germany, 1958.

[24] B. C. Berndt, Ramanujan's Notebooks Part I, Springer Verl., Berlin, Germany, 1985.

[25] B. C. Berndt, Ramanujan's Notebooks Part II, Springer Verl., Berlin, Germany, 1989.

[26] B. C. Berndt, Ramanujan's Notebooks Part IV, Springer Verl., Berlin, Germany, 1994.

[27] B. C. Berndt, "Modular transformations and generalizations of several formulae of Ramanujan," Rocky Mountain Journal of Mathematics, vol. 7, no. 1, pp. 147-190, 1977.

[28] T. Ishii and T. Oda, "A short history on investigation of the special values of zeta and $L$-functions of totally real number fields," in Proceedings of the Conference in Memory of Tsuneo Arakawa, Automorphic Forms and Zeta Functions, World Scientific, Singapore, 2006.

[29] S. Kanemitsu, Y. Tanigawa, and M. Yoshimoto, "Ramanujan's formula and modular forms, number-theoretic methods-future trends," in Proceedings of a Conference Held in Iizuka, S. Kanemitsu and C. Jia, Eds., pp. 159-212, Kluwer, Dordrecht, Netherlands, 2002.

[30] A. Terras, "Some formulas for the Riemann zeta function at odd integer argument resulting from fourier expansions of the Epstein zeta function," Acta Arithmetica, vol. 29, no. 2, pp. 181-189, 1976.

[31] A. Terras, “The fourier expansion of Epstein's zeta function over an algebraic number field and its consequences for algebraic number theory," Acta Arithmetica, vol. 32, no. 1, pp. 37-53, 1977.

[32] P. C. Pasles and W. d. A. Pribitkin, "A generalization of the Lipschitz summation formula and some applications,"
Proceedings of the American Mathematical Society, vol. 129, no. 11, pp. 3177-3185, 2001.

[33] H. M. Srivastava and J.-S. Choi, Series Associated with the Zeta and Related Functions, Kluwer Academic Publishers, Dordrecht, Netherlands, 2001.

[34] X.-H. Wang, S. Kanemitsu, and J. Mehta, "The boundary Lerch zeta-function and short character sums a là Y. Yamamoto," Kyushu Journal of Mathematics, 2020.

[35] A. Erdélyi, W. Magnus, F. Oberhettinger, and F. G. Tricomi, Higher Trancendental Functions, McGraw-Hill, New York, NY, USA, 1953.

[36] E. Grosswald, Topics from the Theory of Numbers, Birkháuser, Basel, Switzerland, 1984.

[37] S. Kanemitsu, M. Katsurada, and M. Yoshimoto, "On the Hurwitz-Lerch zeta-function," Aequationes Mathematicae, vol. 59, no. 1, pp. 1-19, 2000.

[38] H. Rademacher, Topics in Analytic Number Theory, Springer, Berlin, Germany, 1973.

[39] N. S. Koshlyakov, "Investigation of some questions of analytic theory of the rational and quadratic fields, I (Russian)," Izvestiya Akademii Nauk SSSR. Seriya Matematicheskaya, vol. 18, pp. 113-144, 1954.

[40] N. S. Koshlyakov, "Investigation of some questions of analytic theory of the rational and quadratic fields, II (Russian)," Izvestiya Akademii Nauk SSSR. Seriya Matematicheskaya, vol. 18, pp. 213-260, 1954, in Russian.

[41] N. S. Koshlyakov, "Investigation of some questions of analytic theory of the rational and quadratic fields, III (Russian)," Izvestiya Akademii Nauk SSSR. Seriya Matematicheskaya, vol. 18, pp. 307-326, 1954, in Russian.

[42] N. S. Koshlyakov, "Letter to the editor," Izvestiya Akademii Nauk SSSR. Seriya Matematicheskaya, vol. 19, p. 271, 1955 in Russian.

[43] M. Ishibashi and S. Kanemitsu, "Dirichlet series with periodic coefficients," Results in Mathematics, vol. 35, no. 1-2, pp. 70-88, 1999.

[44] W. Schnee, "Die funktionalgleichung der zetafunktion und der dirichletschen reihen mit periodischen koeffizienten," Mathematische Zeitschrift, vol. 31, no. 1, pp. 378-390, 1930.

[45] R. A. Smith, "The average order of arithmetical functions over arithmetic progressions with applications to quadratic forms," Journal für die reine und angewandte Mathematik, vol. 317, pp. 74-87, 1980.

[46] N. L. Wang and S. Banerjee, "On the product of Hurwitz zetafunctions," Proceedings of the Japan Academy, Series A, Mathematical Sciences, vol. 93, no. 5, pp. 31-36, 2017.

[47] E. W. Barnes, "On the theory of multiple gamma function," Transactions of the Cambridge Philosophical Society, vol. 19, pp. 374-425, 1904.

[48] K. Chakraborty, S. Kanemitsu, and T. Kuzumaki, "On the Barnes multiple zeta- and gamma function," Siauliai Mathematical Seminar, vol. 9, pp. 27-41, 2014.

[49] G. H. Hardy and J. E. Littlewood, "Some problems of diophantine approximation: the lattice-points of a right-angled triangle," Proceedings of the London Mathematical Society, vol. s2-20, pp. 212-249, 1922.

[50] G. H. Hardy and J. E. Littlewood, "Some problems of diophantine approximation: the analytic properties of certain Dirichlet's series associated with the distribution of numbers to modulus unity," Transactions of the Cambridge Philosophical Society, vol. 22, pp. 519-533, 1923.

[51] T. Estermann, "On the representations of a number as the sum of two products," Proceedings of the London Mathematical Society, vol. 41, no. 2, pp. 123-133, 1931. 
[52] F. Sato, "List of references on PHV," Surikaiseki-kenkyuusho Kokyuroku, vol. 924, pp. 263-296, 1980.

[53] H. Tsukada, "A general modular relation in analytic number theory. Number theory: sailing on the sea of number theory," Proceedings of the 4th China-Japan Seminar on Number Theory 2006, pp. 214-236, World Scientific, Singapore, 2007. 